\title{
Clinical applications of stem cell therapy - the pros and cons of stem cell sources
}

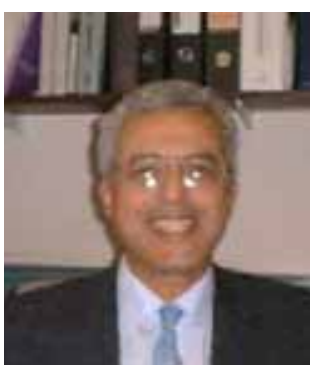

NagyA Habib ${ }^{1+} \&$ M Y Gordon 1

${ }^{\dagger}$ Author for correspondence ${ }^{1}$ Imperial College Faculty of M edicine, D epartments of Surgery and H aematology, $\mathrm{H}$ ammersmith Campus, London, UK

Tel.: +44 208383 8574; Fax: +44 208383 3212;

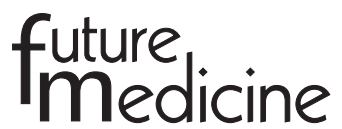

\author{
'...the revolution of using stem cells \\ for regenerative medic ine \\ has begun.'
}

Stem cells with the potential for the treatment of a wide range of degenerative disorders may be obtained from a variety of sources but, for practical reasons, some of them are more likely to find earlier clinical application than others. The main types that have been studied in the context of stem cell therapy are embryonic stem cells, fetal stem cells and adult stem cells.

Embryonic stem cells and, to a lesser extent, fetal stem cells have the potential to repair many types of tissue because they are totipotent [1]. Embryonic stem cells can be greatly increased in number in culture as cell lines in vitro and may be immuno-priviledged. These attributes mean that they can be used to treat multiple patients. H owever, their use has been confounded by serious ethical issues [2] and the very real likelihood that, being immortal, they will form tumors after they have been transplanted into patients [3]. Undoubtedly, however, these barriers to widespread application will be overcome in the future.

Adult stem cells exist in many tissues and organs and these stem cells have differentiation potentials beyond those required to regenerate the tissue or organ in which they reside [4]. Clearly, some of these sources of adult stem cells, like brain stem cells, are less accessible than others, such as bone marrow stem cells. The use of bone marrow-derived stem cells for tissue and organ repair has the additional advantage that there is considerable experience in the clinical application of bone marrow transplantation for the regeneration of the hemopoietic system, dating from the 1970s when relatively large clinical studies were initiated. By 2002, 20,207 hemopoietic stem cell transplants had been performed in Europe by 586 teams in 39 countries. This clinical experience has been accompanied by a wealth of laboratory studies on hemopoietic stem cells and transplantation biology.
In general, two distinct stem cell populations are thought to reside in hemopoietic tissue. These are hemopoietic stem cells and the mesenchymal stem cells. Classically, hemopoietic stem cells are the precursors of all of the blood cell lineages [5] and the mesenchymal stem cells are the source of the supporting stromal cells of the bone marrow [6], including the osteogenic, chondrogenic and adipogenic lineages [7]. Multipotent adult progenitor cells (MAPCs) are a subpopulation of cells that arise in cultures of mesenchymal stem cells and seem to have a broader differentiation potential than the mesenchymal stem cells themselves [8]. H owever, many cell population doublings are required before MAPC S arise in mesenchymal cell cultures and this has been associated with a potential for genetic instability. 0 ther consequences are that it is not known if MAPCs exist in vivo or what their in vivo phenotype may be, M APC s can not be prospectively isolated from a tissue like the bone marrow and there is no quantative assay for MAPC s so that it is not possible to predict with any accuracy how much tissue would be required to supply enough cells for a particular application. Similar considerations apply to other types of stem cell subpopulations with a mesenchymal cell origin that have been described [9-11].

$\mathrm{H}$ ighly desirable features of stem cells for therapy would be the identification of a homogeneous stem cell population that exists normally in vivo and which could be prospectively isolated from an easily available tissue such as the blood or bone marrow. Ideally, prolonged tissue culture would not be necessary and the numbers of cells required would be attainable within a short period of time. Identification of a cell type in the bone marrow with these properties and with the ability to differentiate into multiple cell types would fulfil the immediate requirements for the early clinical application of stem cell therapy. Recently, we found that the hemopoietic stem cell population contains just such a stem cell subpopulation and have used the cells in a Phase I clinical trial for the treatment of liver insufficiency. Results to date are promising and indicate the potential for clinical benefit [12]. 
Adult stem cells may be used in the autologous or allogeneic setting for the treatment of degenerative disorders. Although much has been learnt about tissue typing and matching from clinical tissue and organ transplantation, autologous transplantation avoids the risk of rejection. H owever, without the introduction of allogeneic transplantation, together with the ability to massively expand stem cell numbers in vitro, a single donation is unlikely to be sufficient to supply stem cells for more than one patient. The difficulties associated with amplifying hemopoietic stem cell numbers are notorious because stem cells tend to divide asymmetrically and this is incompatible with an increase in stem cell numbers [13-16]. O nce again, however, large numbers of cell divisions introduce the risk of genetic instability [17]. Some of these obstacles may be overcome by so-called therapeutic cloning although it attracts some of the controversies associated with embryonic stem cell research.

In spite of the limited knowledge about the best source and type of stem cells to use for clin- ical applications, stem cell therapy for degenerative conditions is being applied in several settings. For example, stem cell-induced cardiac regeneration in patients with ischemic heart failure has now been investigated by many groups with encouraging results [18]. The cells administered were obtained from bone marrow and injected by intramyocardial, intracoronary and transendocardial routes. We have performed a Phase I clinical trial of stem cell transplantation in patients with liver insufficiency. For this, autologous mobilized stem cells were procured and purified before they are injected into the portal vein or hepatic artery for local delivery into the damaged tissue [12]. This experience has demonstrated the safety and lack of toxicity of the procedure and has led to the initiation of a Phase II clinical trial. These examples demonstrate that the revolution of using stem cells for regenerative medicine has begun. It is to be anticipated that the future will see ever expanding applications of this novel approach to conditions involving tissue damage and degeneration.

\section{Bibliography}

1. Lerou PH, D aley G Q : Therapeutic potential of embryonic stem cells. Blood Rev. 19, 321-331 (2005).

2. D olgin JL: Embryonic discourse. Issues Law M ed. 19, 203-261 (2004).

3. Erdo F, Buhrle C, Blunk J et al: H ostdependent tumorigenesis of embryonic stem cell transplantation in experimental stroke. J. Cereb. Blood Flow M etab. 23, 780-785 (2003).

4. Lakshmipathy U, Verfaillie C: Stem cell plasticity. Blood Rev. 19, 29-38 (2005).

5. Q uesenberry PJ, Levitt L: $\mathrm{H}$ ematopoietic stem cells. N. Engl J M ed. 301(Pt 1-3) 755-760, 819-823, 868-872 (1979).

6. Friedenstein AJ, Chailakhjan RK, Lalykina KS: The development of fibroblast colonies in monolayers or guinea-pig bone marrow and spleen cells. Cell Tissue Kinet. 3, 393-403 (1970)

7. Pereira $\mathrm{R}, \mathrm{H}$ alford $\mathrm{K}, \mathrm{O} \mathrm{H}^{\mathrm{H}}$ ara $\mathrm{M}$ et al: Cultured adherent cells from marrow can serve as long-lasting precursor cells for bone, cartilage and lung in irradiated mice. Proc. N atl Acad. Sci. U SA 92, 4857-4861 (1995).
8. Colter D C, Sekiya I, Prockop DJ: Identification of a subpopulation of rapidly self-renewing and multipotential adult stem cells in colonies of human marrow stromal cells. Proc. Natl Acad. Sci. U SA 98(14), 7841-7845 (2001).

9. Jiang $Y$, Jahagirder $B N$, Reinhardt RL et al: Pluripotency of meenchymal stem cells derived from adult marrow. $N$ ature 418 , 41-49 (2002).

10. Smith JR, Pochampally R, Perry A, H su S$C$, Prockop D J: Isolation of a highly clonogenic and multipotential subfraction of adult stem cells from bone marrow stroma. Stem Cells 22, 823-831 (2004).

11. Kogler G, Sensken S, Airy JA et al: A new human somatic stem cell from human placental cord blood with intrinsic pluripotent differentiation potential. J. Exp. M ed. 200, 123-135 (2004).

12. Gordon M Y, Levicar N, Bachellier $P$ et al: Characterisation and clinical application of human $\mathrm{CD} 34^{+}$stem/progenitor cell populations mobilised into the blood by $\mathrm{G}$ CSF. Stem Cells Epub ahead of print M ar 23 (2006)
13. Gordon M Y, Blackett N M : Some factors determining the minimum number of cells required for successful clinical engraftment. Bone M arrow Transplant 15, 659-662 (1995).

14. Sherley JL: Asymmetric cell kinetics genes: The key to expansion of adult stem cells in culture. Stem Cells 20, 561-572 (2002).

15. M arley SB, Lewis JL, G ordon M Y: Progenitor cells divide symmetrically to generate new colony-forming cells and clonal heterogeneity. Br. J. H aematol. 121, 643-648 (2003).

16. Joseph N M , M orrison SJ : Towards an understanding of the physiological function of mammalian stem cells. D ev. Cell 9, 173-183 (2005).

17. M iura M, M iura Y, H esed $M$ et al: Accumulated chromosomal instability in murine bone marrow mesenchymal stem cells leads to malignant transformation. Stem Cells24(4), 1095-1103 (2005).

18. D imarakisI, H abib N A, G ordon M Y: Adult bone marrow-derived stem cells and the injured heart: just the beginning? Eur. J. Cardithorac. Surg. 28, 665-676 (2005). 